\title{
SPECTRAL-HOMOTOPY ANALYSIS OF MHD NON-ORTHOGONAL STAGNATION POINT FLOW OF A NANOFLUID
}

\author{
Sarkhosh S. Chaharborij ${ }^{1,2}$, Abbas Moameni ${ }^{1}$ \\ ${ }^{I}$ School of Mathematics and Statistics, Carleton University, Ottawa, KIS 5B6, Canada \\ ${ }^{2}$ Department of Mathematics, Islamic Azad University, Bushehr Branch, Bushehr, Iran \\ saman.seddighi@carleton.ca,momeni@math.carleton.ca
}

Received: 14 May 2017; Accepted: 24 January 2018

\begin{abstract}
In this article, we investigate the theoretical study of the magnetohy-drodynamic (MHD) non-orthogonal stagnation point flow of a nanofluid towards a stretching. The partial differential equations that model the problem are reduced to ordinary differential equations which are then solved analytically using the improved Spectral Homotopy Analysis Method (SHAM). Comparisons of our results from SHAM and numerical solutions show that this method is a capable tool for solving this type of linear and nonlinear problems semi-analytically.
\end{abstract}

MSC 2010: 34B15, 34K07, 34K28

Keywords: nanofluid, MHD stagnation flow, stretching sheet, SHAM

\section{Introduction}

The boundary layer flow problems have various applications in the fluid mechanics. Namely, the classical two-point nonlinear boundary value Blasius problem which models viscous fluid flow over a semi-infinite flat plate, nonlinear Falkner-Skan equation and magnetohydro dynamic (MHD) boundary layer flow.

Most researchers have used the semi-analytical and numerical methods such as the Runge-Kutta methods [1], finite difference methods [2], finite element methods [3] and spectral methods [4] to solve this type of equations. In recent years, for solving nonlinear differential equations, several analytical and semi-analytical methods have been established such as the variational iteration method $[5,6]$, Adomian decomposition method [7], differential transform method [8], homotopy analysis method (HAM) [9-13], and the spectral-homotopy analysis (SHAM) $[14,15]$ and more recently, the successive linearization method $[16,17]$.

All analytical and semi-analytical methods mostly focus on the single and independent linear and nonlinear equations of the boundary layer flow problems. In this paper, we present an improved spectral-homotopy analysis method to solve the system of boundary layer problems. The considered system contains the nonlinear 
boundary differential equations governed from partial differential equations of magnetohy-drodynamic (MHD) non-orthogonal stagnation point flow of a nanofluid towards a stretching.

\section{Formulation of the problem}

We investigate the steady two-dimensional stagnation point flow of a second grade nanofluid over a stretching surface $[18,19]$. Two equal and opposite forces are applied along the z-axis so that the surface is stretched keeping the origin fixed, as shown in Figure 1. We further assume that the surface has temperature $T_{w}$ and the fluid has uniform ambient temperature $T_{\infty}$ (here $T_{w}>T_{\infty}$ ). The flow is subjected to the combined effect of thermal radiation and a transverse magnetic field of strength $B_{0}$, which is assumed to be applied in the positive $y$ direction, normal to the surface. The induced magnetic field is also assumed negligible compared to the applied magnetic field, so it can be neglected. It is further assumed that the base fluid and the suspended nanoparticles are in thermal equilibrium. It is chosen that the coordinate system $x$-axis is along the stretching sheet and $y$-axis is normal to the sheet. Under the above assumptions, the governing equation of the conservation of mass, momentum, energy and nanoparticles fraction in the presence of a magnetic field and thermal radiation past a stretching sheet can be expressed as,

$$
\begin{gathered}
\frac{\partial u}{\partial x}+\frac{\partial v}{\partial y}=0 \\
u \frac{\partial u}{\partial x}+v \frac{\partial u}{\partial y}=-\frac{1}{\rho_{f}} \frac{\partial p}{\partial x}+v\left(\frac{\partial^{2} u}{\partial x^{2}}+\frac{\partial^{2} u}{\partial y^{2}}\right)+\frac{\sigma \beta_{0}^{2}}{\rho} u \\
u \frac{\partial v}{\partial x}+v \frac{\partial v}{\partial y}=-\frac{1}{\rho_{f}} \frac{\partial p}{\partial x}+v\left(\frac{\partial^{2} v}{\partial x^{2}}+\frac{\partial^{2} v}{\partial y^{2}}\right)+\frac{\sigma \beta_{0}^{2}}{\rho} v \\
u \frac{\partial T}{\partial x}+v \frac{\partial T}{\partial y}=\alpha\left(\frac{\partial^{2} T}{\partial x^{2}}+\frac{\partial^{2} T}{\partial y^{2}}\right) \\
+\tau\left[D_{B}\left(\frac{\partial C}{\partial x} \frac{\partial T}{\partial x}+\frac{\partial C}{\partial y} \frac{\partial T}{\partial y}\right)+\frac{D_{T}}{T_{\infty}}\left[\left(\frac{\partial T}{\partial x}\right)^{2}+\left(\frac{\partial T}{\partial y}\right)^{2}\right]\right]-\frac{\partial q_{r}}{\partial y} \\
u \frac{\partial C}{\partial x}+v \frac{\partial C}{\partial y}=D_{B}\left(\frac{\partial^{2} C}{\partial x^{2}}+\frac{\partial^{2} C}{\partial y^{2}}\right)+\left(\frac{D_{T}}{T_{\infty}}\right)\left(\frac{\partial^{2} T}{\partial x^{2}}+\frac{\partial^{2} T}{\partial y^{2}}\right),
\end{gathered}
$$

on $\mathbb{R} \times(0, \infty]$.

Here $u$ and $v$ are the velocity components in the $x$ and $y$ directions. T, $C, v, \rho, \sigma$, and $k$ are the local temperature of the fluid, nano-particle fraction, kinematic viscosity, density, electrical conductivity, and permeability of the saturated porous medium parameters, respectively. $\alpha=K /(\rho c)_{f}$ is the thermal diffusivity, $D_{B}$ is the Brownian motion coefficient, in general the thermal diffusion coefficient $D_{T}$ is a function of temperature and concentration, which complicates the description 
of thermophoresis and $\tau=(\rho c)_{f} /(\rho c)_{p}$ is the ratio of effective heat capacity of the nanoparticle material to heat capacity of the fluid and $\rho_{f}$ is the density of nanofluid at constant pressure. The boundary conditions are,

$$
\begin{gathered}
u=u_{w}(x)=c x, v=0, T=T_{w}, C=C_{w} \text { at } y=0 \\
u=a x \sin \gamma+b y \cos \gamma, v=-a y \sin \gamma, T=T_{\infty}, C=C_{\infty}, y \text { as } y \rightarrow \infty .
\end{gathered}
$$

Where $a$ and $b$, are positive constants with the dimension of (time) ${ }^{-1}$ indicating potential flow and linear shear flow parallel to the streamwise direction (shear stress $b$ ) contributions into the oblique flow, respectively. Radiative heat flux $q_{r}$ in governing boundary layer equation of energy is approximated by Rosseland approximation, which gives,

$$
q_{r}=-\frac{4 \sigma^{*}}{3 k_{1}} \frac{\partial T^{4}}{\partial y}
$$

It is assumed that the temperature difference within the flow is so small that $T^{4}$ can be expressed as a linear function of $T_{\infty}$. This can be obtained by expanding $T^{4}$ in a Taylor series about $T_{\infty}$ and neglecting the higher order terms will results $T^{4} \cong 4 T_{\infty}^{3}-3 T_{\infty}^{4}$. Heat is transferred by forced convection, which involves the only normal component of flow field. Introducing the following dimensionless quantities, the mathematical analysis of the problem is simplified by using similarity transformations.

$$
\xi=(c / v)^{1 / 2} x, \eta=(c / v)^{1 / 2} y, \theta(\eta)=\frac{T-T_{\infty}}{T_{w}-T_{\infty}}, \phi(\eta)=\frac{C-C_{\infty}}{C_{w}-C_{\infty}} .
$$

According to the presented similarity transformations as shown in $[18,19]$, the above systems can be converted to the following ordinary differential equations along with the corresponding boundary conditions,

$$
\begin{gathered}
f^{\prime \prime \prime}+f f^{\prime \prime}-f^{\prime 2}-M^{2}\left(f^{\prime}-\lambda \sin \gamma\right)+\lambda^{2} \sin ^{2} \gamma=0, \\
g^{\prime \prime \prime}+f g^{\prime \prime}-f^{\prime} g^{\prime}-M^{2}\left(g^{\prime}-r \eta \cos \gamma\right)-\alpha r \cos \gamma=0, \\
\left(1+\frac{4}{3} R\right) \theta^{\prime \prime}+\operatorname{Pr}\left(f \theta^{\prime}+N b f^{\prime} \theta^{\prime}+N t \theta^{2}\right)=0, \\
\phi^{\prime \prime}+L e f \phi^{\prime}+(N t / N b) \theta^{\prime \prime}=0,
\end{gathered}
$$

on $(0, \infty]$ and subject to the boundary conditions,

$$
\begin{aligned}
& f(0)=0, f^{\prime}(0)=1, g(0)=0, g^{\prime}(0)=0, \theta(0)=1, \phi(0)=1, \\
& f^{\prime}(\eta) \rightarrow \lambda \sin \gamma, g^{\prime \prime}(\eta)=r \cos \gamma, \theta(\eta) \rightarrow 0, \phi(\eta) \rightarrow 0 \text { as } \eta \rightarrow \infty,
\end{aligned}
$$

where, $\lambda=a / c$ and $r=b / c$ are positive constants and $\gamma$ is striking angle, $M^{2}=\beta_{0}^{2}(\sigma / \rho a)^{1 / 2}$ is the magnetic number, $\operatorname{Pr}=v / \alpha$ is the Prandtl number, 
$R=-\frac{4 \sigma^{*} T_{\infty}^{3}}{k_{1} k}$ is thermal radiation effect, $N t=\tau D_{B}\left(T_{w}-T\right) / T_{\infty} v$ is the thermophoresis parameter, $L e=v / D_{B}$ is the Lewis number.

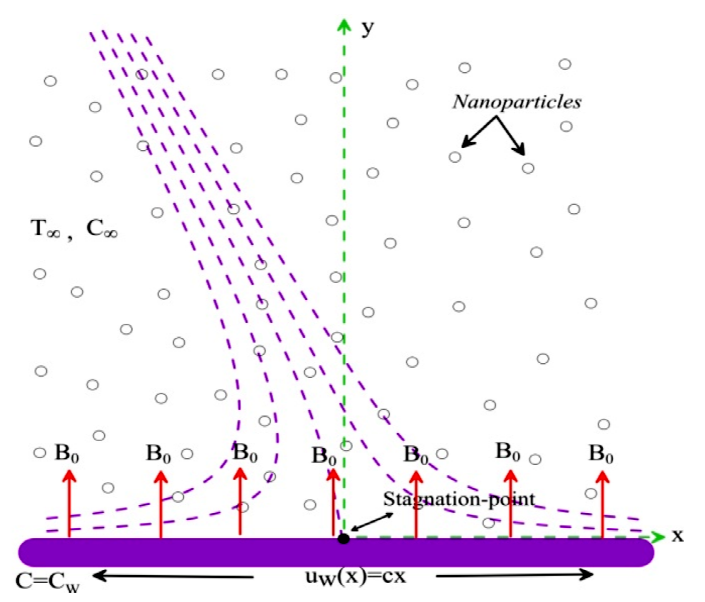

Fig. 1. Geometry of problem

\section{The spectral homotopy analysis method}

At the beginning, for transferring the domain of the problem from $[0, \infty)$ to $[-1,1]$, the domain truncation method has been utilized. The computational domain $[0, L]$ where $L$ is a fixed length can be used to approximate the domain $[0, \infty)$. Here, $L$ is taken to be larger than the thickness of the boundary layer [15]. The simple algebraic mapping for transferring the interval $[0, L]$ to the Chebyshev domain $[-1,1]$ is as follows,

$$
\xi=\frac{2 \eta}{L}-1 ; \xi \in[-1,1] .
$$

For convenience, the boundary conditions have been made homogeneous by applying the transformations as,

$$
\begin{aligned}
& f(\eta)=F(\eta)+f_{0}(\eta), g(\eta)=G(\eta)+g_{0}(\eta), \theta(\eta)=H(\eta)+\theta_{0}(\eta), \phi(\eta)= \\
& \psi(\eta)+\phi_{0}(\eta),
\end{aligned}
$$

where, $f_{0}(\eta), g_{0}(\eta), \theta_{0}(\eta)$ and $\phi_{0}(\eta)$ are chosen so as to satisfy the boundary conditions (6) and (7) as,

$$
\begin{gathered}
f_{0}(\eta)=-\eta e^{-\eta} \lambda \sin (\gamma)+\eta \lambda \sin (\gamma)-e^{-\eta}+1, \\
g_{0}(\eta)=-\eta^{2} e^{-\eta} r \cos (\gamma)+\frac{1}{2} \eta^{2} r \cos (\gamma), \\
\theta_{0}(\eta)=\mathrm{e}^{-\eta}, \phi_{0}(\eta)=e^{-\eta} .
\end{gathered}
$$


Substituting Eqs. (10) into Eqs. (2)-(7) yields that,

$$
\begin{gathered}
\frac{8}{L^{3}} F^{\prime \prime \prime}+\frac{4}{L^{2}} a_{1} F^{\prime \prime}+\frac{4}{L^{2}} F F^{\prime \prime}-2 \frac{M^{2}}{L} F^{\prime}+a_{2} F+a_{3}+a_{4}+a_{5}=0, \\
\frac{8}{L^{3}} G^{\prime \prime \prime}+\frac{4}{L^{2}} b_{1} G^{\prime \prime}-\frac{4}{L^{2}} G^{\prime \prime} F^{\prime}-\frac{4}{L^{2}} G^{\prime} F^{\prime}-\frac{2}{L} b_{2} G^{\prime}-\frac{2}{L} b_{3} F^{\prime}+b_{4} F+b_{5}=0, \\
K H^{\prime \prime}+c_{1} \operatorname{Pr}^{\prime}-c_{2} \operatorname{Pr} F+c_{3} F^{\prime}+4 \frac{N b}{L^{2}} \operatorname{Pr} H^{\prime} F+4 \frac{N t}{L^{2}} \operatorname{Pr}_{H^{\prime}}{ }^{2}+c_{4}=0, \\
\frac{4}{L^{2}} \psi^{\prime \prime}+\frac{2}{L} d_{1} \psi^{\prime}+\frac{2}{L} L e F \psi^{\prime}+16 \frac{N t}{N b L^{4}} H^{\prime 2}+d_{2} H^{\prime \prime}+d_{3} F+d_{4}=0,
\end{gathered}
$$

with the boundary conditions,

$$
\begin{aligned}
& F(-1)=0, F^{\prime}(-1)=0, G(-1)=0, G^{\prime}(-1)=0, H(-1)=0, \psi(-1)=0, \\
& F^{\prime}(1)=0, G^{\prime \prime}(1)=0, H(1)=0, \psi(1)=0,
\end{aligned}
$$

where the coefficients $a_{1}, \ldots, a_{5}$ in Eq. (11) are defined by,

$$
\begin{aligned}
& \ell_{1}=e^{-\frac{1}{2} L(\xi+1)} \lambda \sin (\gamma), \ell_{2}=\frac{1}{2} L(\xi+1) \lambda \sin (\gamma), \ell_{3}=e^{-\frac{1}{2} L(\xi+1)}, \ell_{4}=r \cos (\gamma) \\
& a_{1}=-\frac{1}{2} L(\xi+1) \ell_{1}+\ell_{2}-\ell_{3}+1, a_{2}=2 \ell_{1}-\frac{1}{2} L(\xi+1) \ell_{1}-\ell_{3}, \\
& a_{3}=-3 \ell_{1}+\ell_{2}++\ell_{3}, a_{4}=a_{1} a_{2}, a_{5}=-M^{2}\left(-\ell_{1}+\ell_{2}+\ell_{3}\right)+\lambda^{2} \sin ^{2}(\gamma), \\
& \text { and the coefficients } b_{1}, \ldots, b_{5} \text { in Eq. (12) appear as following: }
\end{aligned}
$$$$
\begin{aligned}
b_{1} & =-\frac{1}{2} L(\xi+1) \ell_{1}+\ell_{2}-\ell_{3}+1, b_{2}=\ell_{3}-\ell_{1}+\frac{1}{2} L(\xi+1) \ell_{1}+\lambda \sin (\gamma)+M^{2}, \\
b_{3} & =-L(\xi+1) \ell_{3} \ell_{4}+\frac{1}{4} L^{2}(\xi+1)^{2} \ell_{3} \ell_{4}+\frac{1}{2} L(\xi+1) \ell_{4}, \\
b_{4} & =\ell_{4}-2 \ell_{3} \ell_{4}+2 L(\xi+1) \ell_{3} \ell_{4}-\frac{1}{4} L^{2}(\xi+1)^{2} \ell_{3} \ell_{4}, \\
b_{5} & =6 \ell_{3} \ell_{4}-3 L(\xi+1) \ell_{3} \ell_{4}+\frac{1}{4} L^{2}(\xi+1)^{2} \ell_{3} \ell_{4}+b_{4}-\left(\ell_{3}-\ell_{1}+\ell_{2}+\lambda \sin (\gamma)\right) \\
& \times\left(-L(\xi+1) \ell_{3} \ell_{4}+\frac{1}{4} L^{2}(\xi+1)^{2} \ell_{3} \ell_{4}+\frac{1}{2} L(\xi+1) \ell_{4}\right) \\
& -M^{2}\left(-L(\xi+1) \ell_{3} \ell_{4}+\frac{1}{4} L^{2}(\xi+1)^{2} \ell_{3} \ell_{4}\right)-\alpha \cos (\gamma),
\end{aligned}
$$

and here are the coefficients $c_{1}, \ldots, c_{4}$ and $d_{1}, \ldots, d_{4}$ in Eqs. (13) and (14) respectively,

$$
\begin{aligned}
c_{1} & =\frac{2}{L}\left(-\frac{1}{2} L(\xi+1) \ell_{1}+\ell_{2}-\ell_{3}+1\right) \frac{2}{L} N b\left(\ell_{3}-\ell_{1}+\frac{1}{2} L(\xi+1) \ell_{1}+\lambda \sin (\gamma)\right) \\
& -\frac{4}{L} N t \ell_{3} \\
c_{2} & =\ell_{3}, c_{3}=-\frac{2}{L} \operatorname{PrNbc}_{2}, c_{4}=\left(1+\frac{4}{3} R\right) c_{2}+\operatorname{Prc}_{2}\left(c_{2} \ell_{2}+\ell_{2}-\ell_{3}+1\right),
\end{aligned}
$$


$-\operatorname{PrNbc}_{2}\left(c_{2}-c_{2} \lambda \sin (\gamma)+c_{2} \ell_{2}+\lambda \sin (\gamma)\right)+\operatorname{PrNtc}_{2}^{2}$,

$d_{1}=L e\left(-\frac{1}{2} L(\xi+1) \ell_{1}+\ell_{2}-\ell_{3}+1\right), d_{2}=\left(8 N t \ell_{3}\right) /\left(N b L^{2}\right)$,

$d_{3}=-L e \ell_{3}, d_{4}=\ell_{3}\left(1-d_{1}+\frac{N t}{N b} \ell_{3}\right)$. tions,

By solving the linear parts of Eqs. (2) up to (5), we can obtain the initial solu-

$$
\begin{gathered}
\frac{8}{L^{3}} F_{0}^{\prime \prime \prime}+\frac{4}{L^{2}} a_{1} F_{0}^{\prime \prime}-2 \frac{M^{2}}{L} F_{0}^{\prime}+a_{2} F_{0}+a_{3}+a_{4}+a_{5}=0, \\
\frac{8}{L^{3}} G_{0}^{\prime \prime \prime}+\frac{4}{L^{2}} b_{1} G_{0}^{\prime \prime}-\frac{2}{L} b_{2} G_{0}^{\prime}-\frac{2}{L} b_{3} F_{0}^{\prime}+b_{4} F_{0}+b_{5}=0, \\
K H_{0}^{\prime \prime}+c_{1} P r H_{0}^{\prime}-c_{2} \operatorname{Pr}_{0}+c_{3} F_{0}^{\prime}+c_{4}=0, \\
\frac{8}{L^{3}} F_{0}^{\prime \prime \prime}+\frac{4}{L^{2}} a_{1} F_{0}^{\prime \prime}-2 \frac{M^{2}}{L} F_{0}^{\prime}+a_{2} F_{0}+a_{3}+a_{4}+a_{5}=0,
\end{gathered}
$$

subject to the boundary conditions,

$F_{0}(-1)=0, F_{0}^{\prime}(-1)=0, G_{0}(-1)=0, G_{0}^{\prime}(-1)=0, H_{0}(-1)=0, \psi_{0}(-1)=0$,

$F_{0}^{\prime}(1)=0, G_{0}^{\prime \prime}(1)=0, H_{0}(1)=0, \psi_{0}(1)=0$.

The Chebyshev pseudo-spectral method has been used to solve Eqs. (16) up to (19). The unknown functions $F_{0}(\xi), G_{0}(\xi), H_{0}(\xi)$ and $\psi_{0}(\xi)$ are aproximated as truncated series of Chebyshev polynomials as follows,

$F_{0}(\xi) \approx F_{0}^{N}\left(\xi_{j}\right)=\sum_{k=0}^{N} \widehat{F_{k}} T_{1, k}\left(\xi_{j}\right), G_{0}(\xi) \approx G_{0}^{N}\left(\xi_{j}\right)=\sum_{k=0}^{N} \widehat{G_{k}} T_{2, k}\left(\xi_{j}\right)$,

$H_{0}(\xi) \approx H_{0}^{N}\left(\xi_{j}\right)=\sum_{k=0}^{N} \widehat{H_{k}} T_{3, k}\left(\xi_{j}\right), \psi_{0}(\xi) \approx \psi_{0}^{N}\left(\xi_{j}\right)=\sum_{k=0}^{N} \widehat{\psi_{k}} T_{4, k}\left(\xi_{j}\right)$,

where $j=0,1, \ldots, N$ and $T_{i, k}(i=1,2,3,4)$ are the Chebyshev polynomials with coefficients $\widehat{F_{k}}, \widehat{G_{k}}, \widehat{H_{k}}$ and $\widehat{\psi_{k}}$ respectively; $\xi_{0}, \xi_{1}, \ldots, \xi_{N}$ are Gauss-Lobatto collocation points defined as, $\xi_{j}=\cos \frac{\pi j}{N}, j=0,1, \ldots, N$.

Derivatives of the functions $F_{0}(\xi), G_{0}(\xi), H_{0}(\xi)$ and $\psi_{0}(\xi)$ at the collocations points are defined as,

$$
\begin{aligned}
\frac{d^{r} F_{0}}{d \xi^{r}} & =\sum_{k=0}^{N} D_{k, j}^{r} F_{0}\left(\xi_{j}\right), \frac{d^{r} G_{0}}{d \xi^{r}}=\sum_{k=0}^{N} D_{k, j}^{r} G_{0}\left(\xi_{j}\right), \frac{d^{r} H_{0}}{d \xi^{r}} \\
& =\sum_{k=0}^{N} D_{k, j}^{r} H_{0}\left(\xi_{j}\right), \frac{d^{r} \psi_{0}}{d \xi^{r}}=\sum_{k=0}^{N} D_{k, j}^{r} \psi_{0}\left(\xi_{j}\right),
\end{aligned}
$$


where $r$ is the order of differentiation and $D$ is the Chebyshev spectral differential matrix with the entries as,

$D_{k, j}=\left(-\frac{1}{2}\right) \frac{q_{k}}{q_{j}} \frac{(-1)^{k+j}}{\sin (\pi(j+k) / 2 N) \sin (\pi(j-k) / 2 N)}, j \neq k$,

$D_{k, k}=\left(-\frac{1}{2}\right) \frac{x_{k}}{\sin ^{2}(\pi k / N)}, k \neq 0, N$ and $D_{0,0}=-D_{N N}=\frac{2 N^{2}+1}{6}$,

with $q_{i}$ is defined as follows,

$q_{i}=\left\{\begin{array}{c}2 \text { for } i=0 \text { or } N \\ 1 \text { for } 1 \leq i \leq N-1,\end{array}\right.$

substituting Eqs. (21) into Eqs. (16)-(19) yields, $A V_{0}=\Phi$, subject to the boundary conditions,

$$
\begin{aligned}
F_{0}\left(\xi_{0}\right) & =0, \sum_{k=0}^{N} D_{0 j} F_{0}\left(\xi_{j}\right)=0, \sum_{k=0}^{N} D_{N j} F_{0}\left(\xi_{j}\right)=0, G_{0}\left(\xi_{0}\right) \\
& =0, \sum_{k=0}^{N} D_{0 j} G_{0}\left(\xi_{j}\right)=0, \sum_{k=0}^{N} D_{N j}^{2} G_{0}\left(\xi_{j}\right)=0, H_{0}\left(\xi_{0}\right) \\
& =H_{0}\left(\xi_{N}\right)=0, \psi_{0}\left(\xi_{0}\right)=\psi_{0}\left(\xi_{N}\right)=0
\end{aligned}
$$

where,

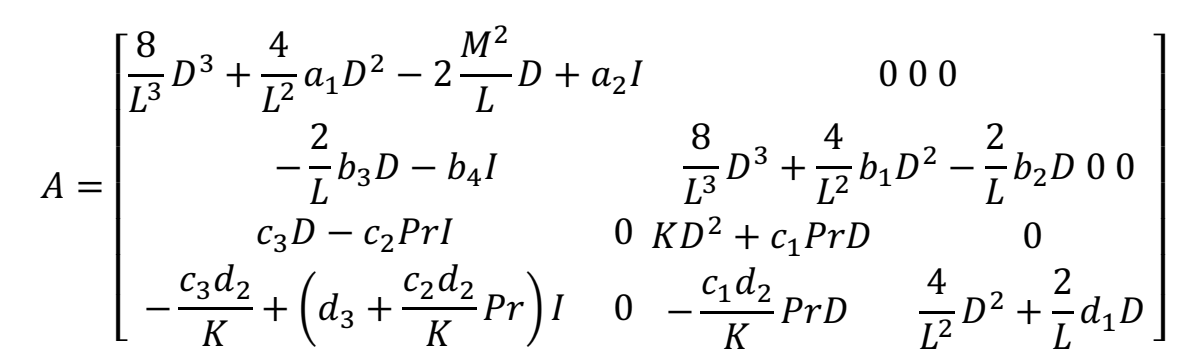

$V_{0}=\left[F_{0}\left(\xi_{0}\right), \ldots, F_{0}\left(\xi_{N}\right), G_{0}\left(\xi_{0}\right), \ldots, G_{0}\left(\xi_{N}\right), H_{0}\left(\xi_{0}\right), \ldots, H_{0}\left(\xi_{N}\right), \psi_{0}\left(\xi_{0}\right), \ldots, \psi_{0}\left(\xi_{N}\right)\right]^{T}$,

$\Phi=\left[\phi_{1}\left(\xi_{0}\right), \ldots, \phi_{1}\left(\xi_{N}\right), \phi_{2}\left(\xi_{0}\right), \ldots, \phi_{2}\left(\xi_{N}\right), \phi_{3}\left(\xi_{0}\right), \ldots, \phi_{3}\left(\xi_{N}\right), \phi_{4}\left(\xi_{0}\right), \ldots, \phi_{4}\left(\xi_{N}\right)\right]^{T}$,

$a_{i}=\operatorname{diag}\left(\left[a_{i}\left(\xi_{0}\right), a_{i}\left(\xi_{1}\right), \ldots, a_{i}\left(\xi_{N-1}\right), a_{i}\left(\xi_{N}\right)\right]\right)$,

$b_{i}=\operatorname{diag}\left(\left[b_{i}\left(\xi_{0}\right), b_{i}\left(\xi_{1}\right), \ldots, b_{i}\left(\xi_{N-1}\right), b_{i}\left(\xi_{N}\right)\right]\right)$,

$c_{i}=\operatorname{diag}\left(\left[c_{i}\left(\xi_{0}\right), c_{i}\left(\xi_{1}\right), \ldots, c_{i}\left(\xi_{N-1}\right), c_{i}\left(\xi_{N}\right)\right]\right)$,

$\phi_{1}=a_{3}+a_{4}+a_{5}, \phi_{2}=a_{5}, \phi_{3}=a_{4}, \phi_{4}=d_{4}, K=(4+16 / 3 R) / L^{2}$.

The superscript $\mathrm{T}$ denotes the transpose, "diag" is a diagonal matrix and $\mathrm{I}$ is an identity matrix of size $(N+1) \times(N+1)$. The values of $\left[V_{0}\left(\xi_{1}\right), V_{0}\left(\xi_{2}\right), \ldots, V_{0}\left(\xi_{N-1}\right)\right]$ are obtained from the equation, $V_{0}=A^{-1} \Phi$, which $V_{0}$ is the initial approximation for the solution of Eqs. (11)-(14) by the SHPM.

The 0 -th order deformation equations are given by,

$(1-p) L_{F}\left[\Omega(\tilde{F}, \tilde{G}, \widetilde{H}, \tilde{\psi})-F_{0}(\xi)\right]=p \hbar_{F}\left\{N_{F}[\tilde{F}(\xi ; p), \tilde{G}(\xi ; p), \widetilde{H}(\xi ; p), \tilde{\psi}(\xi ; p)]-\Gamma_{1}(\xi)\right\}$

$(1-p) L_{G}\left[\Omega(\widetilde{F}, \widetilde{G}, \widetilde{H}, \tilde{\psi})-G_{0}(\xi)\right]=p \hbar_{G}\left\{N_{G}[\tilde{F}(\xi ; p), \widetilde{G}(\xi ; p), \widetilde{H}(\xi ; p), \tilde{\psi}(\xi ; p)]-\Gamma_{2}(\xi)\right\}$ 
$(1-p) L_{H}\left[\Omega(\tilde{F}, \tilde{G}, \widetilde{H}, \tilde{\psi})-H_{0}(\xi)\right]=p \hbar_{H}\left\{N_{H}[\tilde{F}(\xi ; p), \tilde{G}(\xi ; p), \widetilde{H}(\xi ; p), \tilde{\psi}(\xi ; p)]-\Gamma_{3}(\xi)\right\}$ $(1-p) L_{\psi}\left[\Omega(\tilde{F}, \tilde{G}, \widetilde{H}, \tilde{\psi})-\psi_{0}(\xi)\right]=p \hbar_{\psi}\left\{N_{\psi}[\tilde{F}(\xi ; p), \tilde{G}(\xi ; p), \widetilde{H}(\xi ; p), \tilde{\psi}(\xi ; p)]-\Gamma_{4}(\xi)\right\}$ with, $\Omega(\tilde{F}, \tilde{G}, \widetilde{H}, \tilde{\psi})=[\tilde{F}(\xi ; p), \widetilde{G}(\xi ; p), \widetilde{H}(\xi ; p), \tilde{\psi}(\xi ; p)]$. here $H$ is the nonzero convergence controlling auxiliary parameter; $L$ and $N$ are linear and nonlinear operators, respectively, defined as,

$$
\begin{aligned}
& L_{F}[\Omega(\tilde{F}, \tilde{G}, \widetilde{H}, \tilde{\psi})]=\frac{8}{L^{3}} \tilde{F}^{\prime \prime \prime}+\frac{4}{L^{2}} a_{1} \tilde{F}^{\prime \prime}-2 \frac{M^{2}}{L} \tilde{F}^{\prime}+a_{2} \tilde{F}, \\
& L_{G}[\Omega(\tilde{F}, \tilde{G}, \widetilde{H}, \tilde{\psi})]=\frac{8}{L^{3}} \tilde{G}^{\prime \prime \prime}+\frac{4}{L^{2}} b_{1} \tilde{G}^{\prime \prime}-\frac{2}{L} b_{1} \tilde{G}^{\prime}-\frac{2}{L} b_{3} \tilde{F}^{\prime}+b_{4} \tilde{F}, \\
& L_{H}[\Omega(\widetilde{F}, \widetilde{G}, \widetilde{H}, \widetilde{\psi})]=K \widetilde{H}^{\prime \prime}+c_{1} \operatorname{Pr} \widetilde{H}^{\prime}-c_{2} \operatorname{Pr} \widetilde{F}+c_{3} \widetilde{F}^{\prime}, \\
& L_{\psi}[\Omega(\tilde{F}, \tilde{G}, \widetilde{H}, \tilde{\psi})]=\frac{4}{L^{2}} \tilde{\psi}^{\prime \prime}+\frac{2}{L} d_{1} \tilde{\psi}^{\prime}+d_{2} \widetilde{H}^{\prime \prime}+d_{3} \tilde{F}, \\
& N_{F}[\Omega(\tilde{F}, \tilde{G}, \widetilde{H}, \tilde{\psi})]=\frac{8}{L^{3}} \tilde{F}^{\prime \prime \prime}+\frac{4}{L^{2}} a_{1} \tilde{F}^{\prime \prime}+\frac{4}{L^{2}} \tilde{F} \tilde{F}^{\prime \prime}-2 \frac{M^{2}}{L} \tilde{F}^{\prime}+a_{2} \tilde{F}, \\
& N_{G}[\Omega(\tilde{F}, \tilde{G}, \widetilde{H}, \tilde{\psi})]=\frac{8}{L^{3}} \tilde{G}^{\prime \prime \prime}+\frac{4}{L^{2}} b_{1} \tilde{G}^{\prime \prime}+\frac{4}{L^{2}} \tilde{F} \tilde{G}^{\prime \prime}-\frac{4}{L^{2}} \tilde{F}^{\prime} \tilde{G}^{\prime}-\frac{2}{L} b_{1} \tilde{G}^{\prime}-\frac{2}{L} b_{3} \tilde{F}^{\prime}+b_{4} \tilde{F}, \\
& N_{H}[\Omega(\widetilde{F}, \widetilde{G}, \widetilde{H}, \tilde{\psi})]=K \widetilde{H}^{\prime \prime}+c_{1} \operatorname{Pr} \widetilde{H}^{\prime}-c_{2} \operatorname{Pr} \widetilde{F}+c_{3} \widetilde{F}^{\prime}+4 \frac{N b}{L^{2}} \operatorname{Pr} \widetilde{H}^{\prime} \widetilde{F}+4 \frac{N t}{L^{2}} \operatorname{Pr} \widetilde{H}^{\prime 2}, \\
& N_{\psi}[\Omega(\tilde{F}, \tilde{G}, \widetilde{H}, \tilde{\psi})]=\frac{4}{L^{2}} \tilde{\psi}^{\prime \prime}+\frac{2}{L} d_{1} \tilde{\psi}^{\prime}+\frac{2}{L} L e \tilde{F} \tilde{\psi}^{\prime}+16 \frac{N t}{N b L^{4}} \widetilde{H}^{\prime \prime 2}+d_{2} \widetilde{H}^{\prime \prime}+d_{3} \tilde{F} .
\end{aligned}
$$

The $m$-th order deformation equations are given by,

$$
\begin{aligned}
& L_{F}\left[F_{m}(\xi)-\chi_{m} F_{m-1}(\xi)\right]=\hbar_{F} \Re_{m}^{F} L_{G}\left[G_{m}(\xi)-\chi_{m} G_{m-1}(\xi)\right]=\hbar_{G} \Re_{m}^{G}, \\
& L_{H}\left[H_{m}(\xi)-\chi_{m} H_{m-1}(\xi)\right]=\hbar_{H} \Re_{m}^{H}, L_{\psi}\left[\psi_{m}(\xi)-\chi_{m} \psi_{m-1}(\xi)\right]=\hbar_{\psi} \Re_{m}^{\psi},
\end{aligned}
$$

subject to the boundary conditions,

$$
\begin{aligned}
& F_{m}(-1)=F_{m}^{\prime}(-1)=F_{m}^{\prime}(1)=0, G_{m}(-1)=G_{m}^{\prime}(-1)=G_{m}^{\prime \prime}(-1)=0, \\
& H_{m}(-1)=H_{m}(1)=0, \psi_{m}(-1)=\psi_{m}(1)=0,
\end{aligned}
$$

$\chi_{m}=\left\{\begin{array}{l}0, m \leq 1 \\ 1, m>1\end{array}\right.$,

where:

$$
\begin{gathered}
\mathfrak{R}_{m}^{F}(\xi)=\frac{8}{L^{3}} \tilde{F}_{m-1}^{\prime \prime \prime}+\frac{4}{L^{2}} a_{1} \tilde{F}_{m-1}^{\prime \prime}+\sum_{n=0}^{m-1} \frac{4}{L^{2}} \tilde{F}_{n} \tilde{F}_{m-1-n}^{\prime \prime}-2 \frac{M^{2}}{L} \tilde{F}_{m-1}^{\prime}+a_{2} \tilde{F}_{m-1}+\left(1-\chi_{m}\right) \Gamma_{1}, \\
\mathfrak{R}_{m}^{G}(\xi)=\frac{8}{L^{3}} \tilde{G}_{m-1}^{\prime \prime \prime}+\frac{4}{L^{2}} b_{1} \tilde{G}_{m-1}^{\prime \prime}+\sum_{n=0}^{m-1} \frac{4}{L^{2}} \tilde{F}_{n} \tilde{G}_{m-1-n}^{\prime \prime}-\sum_{n=0}^{m-1} \frac{4}{L^{2}} \tilde{F}_{n}^{\prime} \tilde{G}_{m-1-n}^{\prime}-\frac{2}{L} b_{1} \tilde{G}_{m-1}^{\prime} \\
-\frac{2}{L} b_{3} \tilde{F}_{m-1}^{\prime}+b_{4} \tilde{F}_{m-1}+\left(1-\chi_{m}\right) \Gamma_{2},
\end{gathered}
$$




$$
\begin{aligned}
\mathfrak{R}_{m}^{H}(\xi) & =K \widetilde{H}_{m-1}^{\prime \prime}+c_{1} \operatorname{Pr} \widetilde{H}_{m-1}^{\prime}-c_{2} \operatorname{Pr} \widetilde{F}_{m-1}+c_{3} \widetilde{F}_{m-1}^{\prime \prime}+\sum_{n=0}^{m-1} 4 \frac{N b}{L^{2}} \operatorname{Pr} \widetilde{H}_{n}^{\prime} \widetilde{F}_{m-1-n} \\
& +4 \frac{N t}{L^{2}} \operatorname{Pr} \widetilde{H}_{m-1}^{\prime}{ }^{2}+\left(1-\chi_{m}\right) \Gamma_{3} \\
\mathfrak{R}_{m}^{\psi}(\xi) & =\frac{4}{L^{2}} \widetilde{\psi}_{m-1}^{\prime \prime}+\frac{2}{L} d_{1} \widetilde{\psi}_{m-1}^{\prime}+\sum_{n=0}^{m-1} \frac{2}{L} L e \widetilde{F}_{n} \widetilde{\psi}_{m-1-n}^{\prime}+16 \frac{N t}{N b L^{4}} \widetilde{H}_{m-1}^{\prime}{ }^{2}+d_{2} \widetilde{H}_{m-1}^{\prime \prime} \\
& +d_{3} \widetilde{F}_{m-1}+\left(1-\chi_{m}\right) \Gamma_{4} .
\end{aligned}
$$

Applying the Chebyshev pseudospectral transformation to Eqs. (25)-(27) gives,

$$
A V_{m}=\left(\chi_{m}+\hbar\right) A V_{m-1}-\hbar\left(1-\chi_{m}\right) \Phi+\hbar Q_{m-1},
$$

subject to the boundary conditions:

$$
\begin{gathered}
F_{m}\left(\xi_{0}\right)=0, \sum_{k=0}^{N} D_{0 j} F_{m}\left(\xi_{j}\right)=0, \sum_{k=0}^{N} D_{N j} F_{m}\left(\xi_{j}\right)=0, \\
G_{m}\left(\xi_{0}\right)=0, \sum_{k=0}^{N} D_{0 j} G_{m}\left(\xi_{j}\right)=0, \sum_{k=0}^{N} D_{N j}^{2} G_{m}\left(\xi_{j}\right)=0, \\
H_{m}\left(\xi_{0}\right)=H_{m}\left(\xi_{N}\right)=0, \psi_{m}\left(\xi_{0}\right)=\psi_{m}\left(\xi_{N}\right)=0
\end{gathered}
$$

where $\Phi$ are defined in Eq. (24) and

$$
\left.\begin{array}{r}
V_{m}=\left[F_{m}\left(\xi_{0}\right), \ldots, F_{m}\left(\xi_{N}\right), G_{m}\left(\xi_{0}\right), \ldots, G_{m}\left(\xi_{N}\right), H_{m}\left(\xi_{0}\right), \ldots, H_{m}\left(\xi_{N}\right), \psi_{m}\left(\xi_{0}\right), \ldots, \psi_{m}\left(\xi_{N}\right)\right]^{T}, \\
\sum_{m-1}^{m-1} \frac{4}{L^{2}} \tilde{F}_{n} \tilde{F}_{m-1-n}^{\prime \prime} \\
Q_{n=0}^{m-1} \frac{4}{L^{2}} \tilde{F}_{n} \tilde{G}_{m-1-n}^{\prime \prime}-\sum_{n=0}^{m-1} \frac{4}{L^{2}} \tilde{F}_{n}^{\prime} \tilde{G}_{m-1-n}^{\prime} \\
0 \\
\sum_{n=0}^{m-1} \frac{2}{L} L e \tilde{F}_{n} \tilde{\psi}_{m-1-n}^{\prime}+16 \frac{N t}{N b L^{4}} \widetilde{H}_{m-1}^{\prime}{ }^{2}
\end{array}\right]
$$

Boundary conditions (29) are implemented in matrix $A$ on the left side of (28) in rows $1, N, N+1, N+2$ and $2(N+1)$, respectively. Matrix $A$ in the right-hand side of Eq. (28), $\Phi$ and $Q_{m-1}$ have corresponding rows and all columns equal to zero. This recursive formula when $m \geq 1$, can be written as follows,

$$
V_{m}=\left(\chi_{m}+\hbar\right) A^{-1} A V_{m-1}-\hbar A^{-1}\left[\left(1-\chi_{m}\right) \Phi+Q_{m-1}\right] .
$$


Therefore, the higher-order approximation $V_{m}$ for $m \geq 1$ has been obtained by starting from the initial approximation.

\section{Optimization of convergence-control parameters based on the square residual errors}

From Eqs. (27), error functions can be defined as follows:

$\operatorname{Error}_{\mathrm{F}} \approx \mathfrak{R}_{N+1}^{F}(\xi), \operatorname{Error}_{\mathrm{G}} \approx \mathfrak{R}_{N+1}^{G}(\xi), \operatorname{Error}_{\mathrm{H}} \approx \mathfrak{R}_{N+1}^{H}(\xi), \operatorname{Error}_{\psi} \approx \mathfrak{R}_{N+1}^{\psi}(\xi)$.

In what follows, we are going to find the optimal values of $\hbar_{F}, \hbar_{G}, \hbar_{H}$ and $\hbar_{\psi}$, by using the convergence-control for various values of order $N$ will find. The square residual error (SRE) method can help us to optimize the convergence-control parameters as follows:

$$
\begin{aligned}
& \operatorname{SRE}_{\hbar_{\mathrm{F}}}=\int_{\Omega}\left(\text { Error }_{\mathrm{F}}\right)^{2} d \Omega, \mathrm{SRE}_{\hbar_{\mathrm{G}}}=\int_{\Omega}\left(\text { Error }_{\mathrm{G}}\right)^{2} d \Omega, \\
& \mathrm{SRE}_{\hbar_{\mathrm{H}}}=\int_{\Omega}\left(\text { Error }_{\mathrm{H}}\right)^{2} d \Omega, \mathrm{SRE}_{\hbar_{\psi}}=\int_{\Omega}\left(\text { Error }_{\psi}\right)^{2} d \Omega,
\end{aligned}
$$

where Error $_{\mathrm{F}}$, Error $_{\mathrm{G}}$, Error $_{\mathrm{H}}$ and Error $_{\psi}$ are the $N$ th-order SHAM approximations of the functions $F, G, H$ and $\psi$, respectively. Obviously, when $N \rightarrow \infty$ then we will have $\mathrm{SRE}_{\hbar_{\mathrm{F}}} \rightarrow 0, \mathrm{SRE}_{\hbar_{\mathrm{G}}} \rightarrow 0, \mathrm{SRE}_{\mathrm{H}} \rightarrow 0$ and $\mathrm{SRE}_{\hbar_{\psi}} \rightarrow 0$ that correspond to convergent series solutions of SHAM. The points at which the gradient of square residual error functions presented in Eqs. (32) and (33) with respect to convergence-control parameters vanish, are precisely the optimal values of $\hbar_{F}, \hbar_{G}, \hbar_{H}$ and $\hbar_{\psi}$ for $N$ th-order SHAM approximation as follows:

$$
\frac{\partial \mathrm{SRE}_{\hbar_{\mathrm{F}}}}{\partial \hbar_{\mathrm{F}}}=0, \frac{\partial \mathrm{SRE}_{\hbar_{\mathrm{G}}}}{\partial \hbar_{\mathrm{G}}}=0, \frac{\partial \mathrm{SRE}_{\hbar_{\mathrm{H}}}}{\partial \hbar_{\mathrm{H}}}=0, \frac{\partial \mathrm{SRE}_{\hbar_{\Psi}}}{\partial \hbar_{\Psi}}=0,
$$

Figure 2 presents the optimal values $\hbar$ and the optimal square residual errors in the $\hbar\left(\mathrm{SRE}_{\hbar}\right)$ at different values of $N$. This figure shows that the optimal values of $\hbar$ is approximately between -1 and -0.4 . By choosing $\hbar$ from this optimal interval, the results obtained from SHAM will have good accuracy. Table 1 presents the optimal values of $\hbar_{F}, \hbar_{G}, \hbar_{H}$ and $\hbar_{\psi}\left(\hbar=\operatorname{optimal}\left\{\hbar_{F}, \hbar_{G}, \hbar_{H}, \hbar_{\psi}\right\}\right)$ and optimal values of the square residual error at a different order of $N$ in the $\hbar$. Table 1 is showing that with increasing the order of approximation $(N)$, the square residual error will decrease. To obtain the results of Table 1, Matlab R2015b software has been used. 


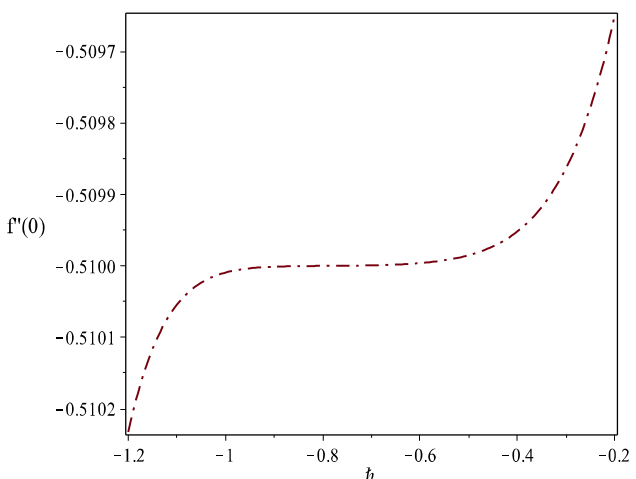

Fig. 2. Optimal $\hbar$-curve for the function $f^{\prime \prime}(0)$ when $M=1, \lambda=1, L e=P r=R=1$, $N t=N b=0.1$ and $\alpha=r=1 / 2$

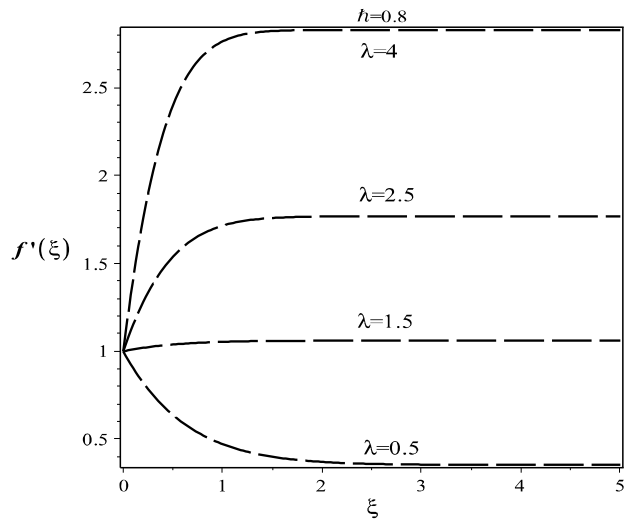

Fig. 4. Influence of $\lambda$ on $f^{\prime}$ when $M=1$, $\gamma=\pi / 4, L e=P r=R=1, N t=N b=0.1$ and $\alpha=r=1 / 2$

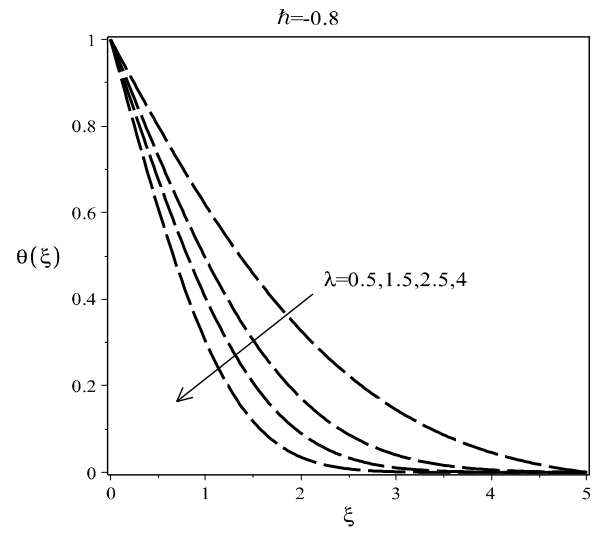

Fig. 6. Influence of $\lambda$ on temperature profile when $M=1, \gamma=\pi / 4, L e=\operatorname{Pr}=R=1$, $N t=N b=0.1$ and $\alpha=r=1 / 2$

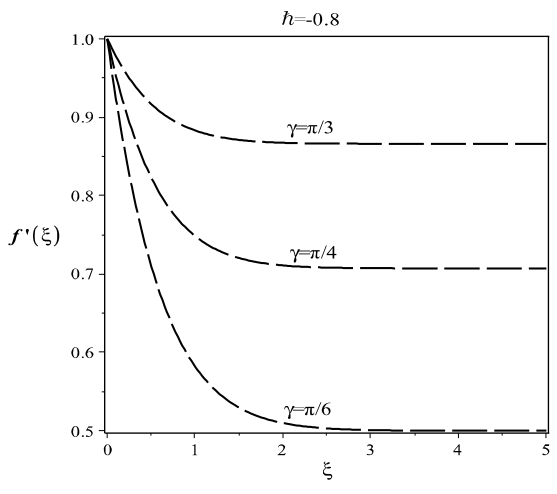

Fig. 3. Influence of $\gamma$ on $f^{\prime}$ when $M=1, \lambda=1, L e=\operatorname{Pr}=R=1$, $N t=N b=0.1$ and $\alpha=r=1 / 2$

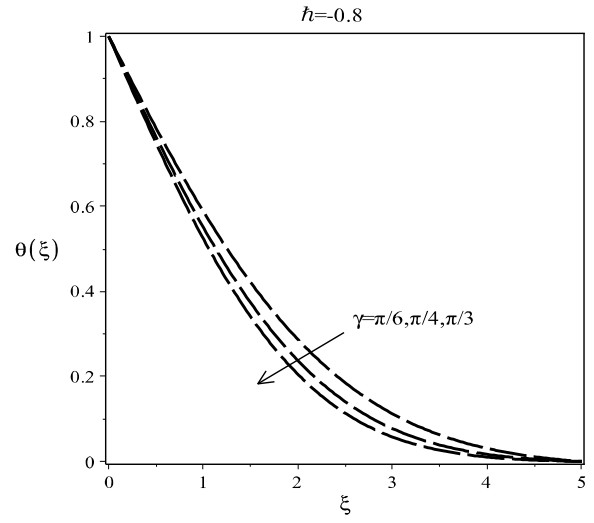

Fig. 5. Influence of $\gamma$ on temperature profile when $M=1, \lambda=1, L e=\operatorname{Pr}=R=1$, $N t=N b=0.1$ and $\alpha=r=1 / 2$

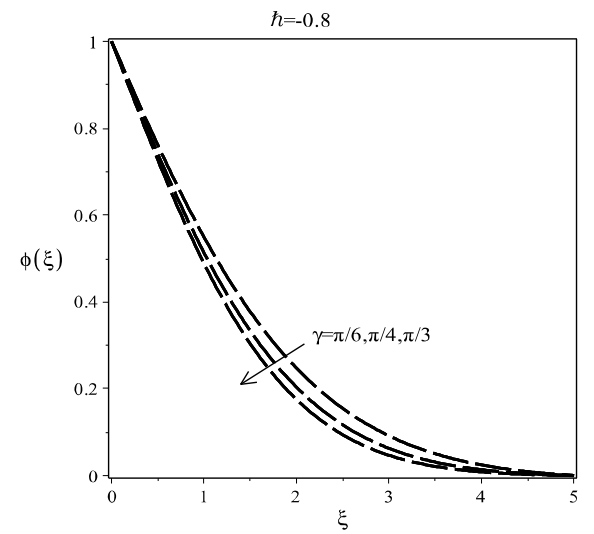

Fig. 7. Influence of $\gamma$ on concentration profile when $M=1, \lambda=1, L e=\operatorname{Pr}=R=1$, $N t=N b=0.1$ and $\alpha=r=1 / 2$ 


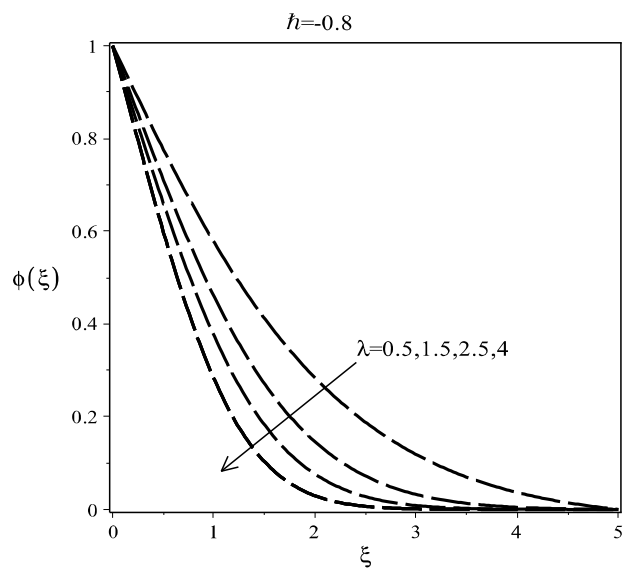

Fig. 8. Influence of $\lambda$ on concentration profile when $M=1, \gamma=\pi / 4, L e=\operatorname{Pr}=R=1$, $N t=N b=0.1$ and $\alpha=r=1 / 2$
The optimal values $\hbar$ and optimal square residual error in $\hbar\left(\mathbf{S R E}_{\hbar}\right)$ at different values of order $N$

\begin{tabular}{|c|c|c|}
\hline $\boldsymbol{N}$ & $\hbar$ & $\mathbf{S R E}_{\hbar}$ \\
\hline 2 & -1.163 & $3.0631 \times 10^{-2}$ \\
\hline 3 & -1.062 & $1.6270 \times 10^{-4}$ \\
\hline 4 & -0.691 & $2.9531 \times 10^{-5}$ \\
\hline 5 & -0.671 & $1.0879 \times 10^{-5}$ \\
\hline 6 & -0.526 & $6.0836 \times 10^{-6}$ \\
\hline 7 & -0.603 & $4.0297 \times 10^{-7}$ \\
\hline 8 & -0.692 & $3.4792 \times 10^{-8}$ \\
\hline 9 & -0.717 & $1.7738 \times 10^{-10}$ \\
\hline 10 & -0.754 & $5.6251 \times 10^{-12}$ \\
\hline 11 & -0.771 & $2.0692 \times 10^{-13}$ \\
\hline 12 & -0.818 & $6.2957 \times 10^{-14}$ \\
\hline
\end{tabular}

\section{Results and discussion}

Figures 3, 5 and 7 present the influence of $\gamma$ on $f^{\prime}$ and, temperature and concentration profiles when $M=1, \lambda=1, L e=P r=R=1, N t=N b=0.1$ and $\alpha=r=$ $=1 / 2$. As shown, by decreasing the values of $\gamma$ appeared in the initial conditions $f^{\prime}(\eta) \rightarrow \lambda \sin \gamma$ and $g^{\prime \prime}(\eta) \rightarrow r \cos \gamma$ from $\pi / 3$ to $\pi / 6$ the profile $f^{\prime}$ is decreasing and both temperature and concentration profiles $\theta$ and $\phi$ are increasing. The results indicate that the curves reduce with a negative slope for $\lambda \sin \gamma<1$. Figure 3 is showing that with decreasing the striking angle $(\gamma)$ at a fixed value of $\xi$, heat transfer velocity will decrease. Figure 5 indicates that with decreasing the striking angle at a fixed value of $\xi$, temperature will increase. Figure 7 is showing that with decreasing the striking angle at a fixed value of $\xi$, heat transfer concentration will increase.

Figures 4, 6 and 8 are showing the influence of $\lambda$ on $f^{\prime}$ and, temperature and concentration profiles when $M=1, \gamma=\pi / 4, L e=\operatorname{Pr}=R=1, N t=N b=0.1$ and $\alpha=r=1 / 2$. Note that, by increasing the values of $\lambda$ appeared in the initial condition $f^{\prime}(\eta) \rightarrow \lambda \sin \gamma$ form 0.5 to 4 the profile $f^{\prime}$ is increasing and both temperature and concentration profiles $\theta$ and $\phi$ are decreasing. Figures 4 indicate, however, that they have positive slopes for $\lambda \sin \gamma<1$, and the curves reduce with a negative slope for $\lambda \sin \gamma<1$. Figure 4 is showing that with increasing the velocity parameter $(\lambda)$ at a fixed value of $\xi$, heat transfer velocity will increase. Figure 6 indicates that with increasing the velocity parameter at a fixed value of $\xi$, temperature will increase. Figure 8 is showing that with increasing the velocity parameter at a fixed value of $\xi$, heat transfer concentration will increase.

In order to test the accuracy of the present results, we have compared the results for skin friction coefficient $f^{\prime \prime}(\eta)$ with those reported by Lok et al. [20]. This 
comparison is presented in Table 2. It is noticed that this comparison shows an excellent agreement, so that we are confident that the present results are accurate. Table 2 shows a comparison of the results $f^{\prime \prime}(0)$ for for $M=0$ at different values of $\lambda$ and $\gamma$. Results of this table indicate that with increasing parameter $\lambda$ from 0.2 to 3.0, for any values of parameter $\gamma, f^{\prime \prime}(0)$ is increasing. As seen, by increasing $\gamma$ from $\pi / 12$ to $\pi / 2$, the function $f^{\prime \prime}(0)$ is increasing with a high speed. To obtain the results of Table 2, Matlab R2015b software has been used.

Comparison of the results for $f^{\prime \prime}(0)$ for $M=0$ at different values of $\lambda$ and $\gamma$.

With $N=12$ and $\hbar=-0.818$

\begin{tabular}{|c|c|c|c|c|c|c|c|c|}
\hline \multirow{2}{*}{$\lambda$} & \multicolumn{2}{|c|}{$\gamma=\pi / 12$} & \multicolumn{2}{c|}{$\gamma=\pi / 4$} & \multicolumn{2}{c|}{$\gamma=\pi / 3$} & \multicolumn{2}{c|}{$\gamma=\pi / 2$} \\
\cline { 2 - 9 } & Present & $\begin{array}{c}\text { Lok et al. } \\
{[20]}\end{array}$ & Present & $\begin{array}{c}\text { Lok et al. } \\
{[20]}\end{array}$ & Present & $\begin{array}{c}\text { Lok et al. } \\
{[20]}\end{array}$ & Present & $\begin{array}{c}\text { Lok et al. } \\
{[20]}\end{array}$ \\
\hline 0.2 & -0.987616 & -0.987032 & -0.950564 & -0.950424 & -0.933744 & -0.933660 & -0.918165 & -0.918110 \\
\hline 0.5 & -0.956437 & -0.956268 & -0.806205 & -0.806205 & -0.734439 & -0.734444 & -0.667265 & -0.667271 \\
\hline 1.0 & -0.879693 & -0.879674 & -0.424309 & -0.424315 & -0.205021 & -0.205025 & - & - \\
\hline 2.0 & -0.648607 & -0.648613 & 0.738447 & 0.738474 & 1.400957 & 1.401023 & 2.017503 & 2.017615 \\
\hline 3.0 & -0.331931 & -0.331937 & 2.313007 & 2.313144 & 3.566349 & 3.566614 & 4.729282 & 4.729694 \\
\hline
\end{tabular}

\section{Conclusion}

We have investigated the modified spectral homotopy analysis method (SHAM) for solving a complicated nonlinear dynamical system in the MHD non-orthogonal stagnation point flow of a nanofluid towards a stretching. Numerical results show the effectiveness of our proposed method for solving complicated linear and nonlinear dynamical systems in the heat transfer and heat flow problems. The results indicate that they have positive slopes for $\lambda \sin \gamma<1$, and the curves reduce with a negative slope for $\lambda \sin \gamma<1$. The optimal interval for $\hbar$ is between -1 and -0.4 . The results for skin friction coefficient $f^{\prime \prime}(\eta)$ from our presented method with $N=12$ and $\hbar=-0.818$ have been compared with those reported by Lok et al. at references [20]. This comparison shows an excellent agreement, so that we are confident that the present results are accurate.

\section{References}

[1] Chaharborj, S.S., Kiai, S.S., Bakar, M.A., Ziaeian, I., \& Fudziah, I. (2012). New impulsional potential for a paul ion trap. International Journal of Mass Spectrometry, 309, 63-69.

[2] Nayak, I., Nayak, A.K., \& Padhy, S. (2016). Implicit fnite difference solution for the magnetohydro-dynamic unsteady free convective flow and heat transfer of a third-grade fluid past a porous vertical plate. International Journal of Mathematical Modelling and Numerical Optimisation, 7(1), 4-19. 
[3] John, V. (2016). Finite Element Methods for Incompressible Flow Problems. Berlin: Erscheint demnchst bei Springer.

[4] Chen, S., \& Wang, Y. (2016). A rational spectral collocation method for third-order singularly perturbed problems. Journal of Computational and Applied Mathematics, 307, 93-105.

[5] Moameni, A. (2011). Non-convex self-dual lagrangians: New variational principles of symmetric boundary value problems. Journal of Functional Analysis, 260(9), 2674-2715.

[6] Wazwaz, A.M. (2016). Solving systems of fourth-order emden-fowler type equations by the variational iteration method. Chemical Engineering Communications, 203(8), 1081-1092.

[7] Hosseini, S., Babolian, E., \& Abbasbandy, S. (2016). A new algorithm for solving van der pol equation based on piecewise spectral adomian decomposition method. International Journal of Industrial Mathematics, 8(3), 177-184.

[8] Fidanoglu, M., Komurgoz, G., \& Ozkol, I. (2016). Heat transfer analysis of fins with spine geometry using differential transform method. International Journal of Mechanical Engineering and Robotics Research, 5(1), 67-71.

[9] Shahlaei-Far, S., Nabarrete, A., \& Balthazar, J.M. (2016). Homotopy analysis of a forced nonlinear beam model with quadratic and cubic nonlinearities. Journal of Theoretical and Applied Mechanics, 54(4), 1219-1230.

[10] Semary, M.S., \& Hassan, H.N. (2016). The homotopy analysis method for q-difference equations. Ain Shams Engineering Journal, DOI http://dx.doi.Org/10.1016/j.asej.2016.02.005.

[11] Hayat, T., Mumtaz, M., Shafiq, A., \& Alsaedi, A. (2017). Stratified magnetohydrodynamic flow of tangent hyperbolic nanofluid induced by inclined sheet. Applied Mathematics and Mechanics, 38(2), 271-288.

[12] Zhu, J., Wang, S., Zheng, L., \& Zhang, X. (2017). Heat transfer of nanofluids considering nanoparticle migration and second-order slip velocity. Applied Mathematics and Mechanics, $38(1), 125-136$.

[13] Zhao, Q., Xu, H., Tao, L., Raees, A., \& Sun, Q. (2016). Three-dimensional free bio-convection of nanofluid near stagnation point on general curved isothermal surface. Applied Mathematics and Mechanics, 37(4), 417-432

[14] Motsa, S.S., Marewo, G.T., Sibanda, P., \& Shateyi, S. (2011). An improved spectral homotopy analysis method for solving boundary layer problems. Boundary Value Problems, 2011(1), 3-11.

[15] Rashidi, M.M., Rostami, B., Freidoonimehr, N., \& Abbasbandy, S. (2014). Free convective heat and mass transfer for MHD fluid flow over a permeable vertical stretching sheet in the presence of the radiation and buoyancy effects. Ain Shams Engineering Journal, 5(3), 901-912.

[16] Bhatti, M.M., Shahid, A., \& Rashidi, M.M. (2016). Numerical simulation of fluid flow over a shrinking porous sheet by Successive linearization method. Alexandria Engineering Journal, 55(1), 51-56.

[17] Ahmed, M.A.M., Mohammed, M.E., \& Khidir, A.A. (2015). On linearization method to MHD boundary layer convective heat transfer with low pressure gradient. Propulsion and Power Research, 4(2), 105-113.

[18] Rashidi, M.M., Abelman, S., \& Mehr, N.F. (2013). Entropy generation in steady MHD flow due to a rotating porous disk in a nanofluid. International Journal of Heat and Mass Transfer, 62, 515-525.

[19] Rashidi, M.M., Ali, M., Freidoonimehr, N., \& Nazari, F. (2013). Parametric analysis and optimization of entropy generation in unsteady MHD flow over a stretching rotating disk using artificial neural network and particle swarm optimization algorithm. Energy, 55, 497-510.

[20] Lok, Y.Y., Amin, N., \& Pop, I. (2006). Non-orthogonal stagnation point flow towards a stretching sheet. International Journal of Non-Linear Mechanics, 41(4), 622-627. 